\title{
VCAM-1 Values in Obese Adolescents with Dyslipidemia and Insulin Resistance
}

\author{
Suhasta Nova*, Roedi Irawan, Nur Aisiyah Widjaja, Irwanto \\ Department of Child Health, Faculty of Medicine Universitas Airlangga, \\ Dr. Soetomo General Academic Hospital, Surabaya, Indonesia
}

*Corresponding author details: Suhasta Nova; suhastanova@yahoo.com

\begin{abstract}
Background: The incidence of obesity in adolescents in the world is increasing. Obesity can lead to atherosclerosis, which is initially accompanied by endothelial dysfunction.

Methods: This study cros sectional study conducted from Februari to September 2021 in Surabaya, indonesia. VCAM-1 was examined in the Institute Tropical Diseases (ITD) laboratory using ELISA methods.

Result: One hundred and sixteen children are included in this study, consisting of 107 obese children and 9 nonobese children. The VCAM-1 values in obese adolescents increased 2.4x compared to those in non-obese adolescents ( $p$-value 0.001). The VCAM-1 values in obese adolescents without dyslipidemia nor insulin resistance increased 2.4x (p-value 0.001), in obese adolescents who had dyslipidemia it increased 2.2x ( $\mathrm{p}$-value 0.001), in obese adolescents who had insulin resistance it increased 1.8x (p-value 0.007), and in obese adolescents who had dyslipidemia and insulin resistance it increased $2.5 \mathrm{x}$ ( $\mathrm{p}$-value 0.007 ) compared to those in non-obese adolescents without dyslipidemia nor insulin resistance. There was no significant difference in the VCAM-1 values neither in obese adolescents who had dyslipidemia (p-value 0.377 ), in obese adolescents who had insulin resistance ( $p$ value 0.174 ), nor obese adolescents who had dyslipidemia and insulin resistance ( $p$-value 0.769) compared to those in obese adolescents who had no dyslipidemia nor insulin resistance.

Conclusion: Obese adolescents who had dyslipidemia and insulin resistance significantly associate to an increase in the VCAM-1 values.
\end{abstract}

Keywords: obese adolescent; dyslipidemia; insulin resistance; VCAM-1

\section{INTRODUCTION}

The world is currently facing an increasing number of obesity cases in children. The increased obesity cases in adolescents lead to increased dyslipidemia and insulin resistance cases, resulting in increased cardiovascular diseases. Cardiovascular diseases are characterized by atherosclerosis in the blood vessels, which the occurrence of endothelial dysfunction will precede [1], [2].

Available data in America shows that $22.8 \%$ of obesity cases occur at the age of $2-5$ years, $34.2 \%$ at the age of $6-11$ years, $34.5 \%$ at the age of $12-19$ years, and $17 \%$ of children aged 2-11 years are obese [3], [4]. Obesity cases in Indonesia have an increasing trend $(10.5 \%$ in $2007,14.18 \%$ in 2013 , and $21.8 \%$ in 2018) [5]. Several studies have shown the prevalence of dyslipidemia in obese children, among others, with a percentage of $45.8 \%$ in Iran, $40 \%$ in Germany, $63 \%$ in India [6]. Another study states that $43 \%$ of obese children had dyslipidemia, and approximately $52.5 \%$ of them had insulin resistance [6], [7]. Besides, approximately $12.5 \%$ of obese children experience a combination of insulin resistance and dyslipidemia [8]. The Bogalusa Heart Study found that approximately $50 \%$ of fatty streak cases accompanied by dyslipidemia occur during childhood, and around $80 \%$ of which occur during adolescence, and the prevalence of fibrous plaque increased from $8 \%$ in childhood to $70 \%$ in youth [9].

Endothelial dysfunction can be triggered by several factors, including obesity, dyslipidemia, insulin resistance, and hypertension [1], [2], [10]. Endothelial dysfunction can be identified by examining several markers, including vascular cell adhesion molecule-1 (VCAM-1), intracellular adhesion molecule -1 (ICAM-1), and e selectin [1], [2]. However, VCAM-1 has a more significant role in the development of early atherosclerosis characterized by endothelial dysfunction compared to other markers [11].

Studying the central role of the endothelium in the development of atherosclerosis is essential not only for understanding its pathophysiology but also for early detection, stratification of cardiovascular risk, and assessment of response to treatment [12]. Studies on VCAM-1 associated with obesity, dyslipidemia, and insulin resistance in Indonesia are still relatively rare. Therefore, it requires further studies of clinical importance for patients at risk for atherosclerosis.

\section{MATERIALS AND METHODS}

This cross-sectional study was conducted from February to September 2021 by examining VCAM-1 in the ITD laboratory. It is a continuation of the previous study with a total sample of 339 (male and female) consisting of 269 obese children and 70 non-obese children. Some nonobese adolescents were unwilling to participate in the study because they thought they were healthy. The sample consisted of 116 male participants. In this study, a total sample of 116 male participants was taken. The sample consisted of 9 non-obese male adolescents and 107 obese male adolescents. The VCAM-1 was examined using an ELISA kit. The data were analyzed using SPSS version 18. The statistical analysis applied the Mann-Whitney test and Spearman test with $\mathrm{CI}>95 \%$ and $\mathrm{P}<0.05$. 


\section{RESULTS}

TABLE 1: Basic characteristics of subjects

\begin{tabular}{ll}
\hline \multicolumn{1}{c}{ Basic Characteristics } & n (\%) \\
\hline Age & \\
\hline Age $<15$ years & $47(41.2 \%)$ \\
Age $\geq 15-18$ years & $67(58.8 \%)$ \\
\hline Gender & $114(100 \%)$ \\
\hline Male & \\
\hline Risk Factors & $8(7 \%)$ \\
Not obese & $106(93 \%)$ \\
Obese & $72(63.2 \%)$ \\
Dyslipidemia & $56(49.15)$ \\
Insulin resistance & $47(41.2 \%)$ \\
Dyslipidemia and insulin resistance & $8(7 \%)$ \\
Not obese, not dyslipidemia, not insulin resistance & $26(22.8 \%)$ \\
Obese, not dyslipidemia, not insulin resistance & $24(21.1 \%)$ \\
Obese, dyslipidemia & $9(7.9 \%)$ \\
Obese, insulin resistance & $47(41.2 \%)$ \\
\hline
\end{tabular}

In this study, $93 \%$ of the subjects were obese adolescents. Most of the subjects in this study were adolescents aged $\geq 15-18$ years, and all of them were male.

TABLE 2: Correlation between Age, Weight, Height, and BMI and VCAM-1

\begin{tabular}{lccc}
\hline \multicolumn{1}{c}{ Basic Characteristics } & Mean/SD & VCAM-1 (Mean/SD) & P $<0.05$ \\
\hline Age & $15.22 / 16.68$ & $13.52 / 15.91$ & $0.509^{*}$ \\
Weight & $85.75 / 15.64$ & & $0.456^{*}$ \\
Height & $164.42 / 7.64$ & & $0.153^{*}$ \\
BMI & $31.51 / 5.02$ & & $0.148^{*}$ \\
\hline
\end{tabular}

(*) Spearman Test

This study revealed that age, weight, height, and BMI did not correlate with the VCAM-1 values.

TABLE 3: Association Between Obese Adolescents who had Dyslipidemia and Insulin Resistance and the VCAM-1 Values

\begin{tabular}{|c|c|c|c|}
\hline Risk Factors & $\mathbf{n}$ & VCAM-1 (Median) & $\mathrm{P}<0.05$ \\
\hline Obese & 106 & 11.27 & \multirow{2}{*}{$0.001^{*}$} \\
\hline Not obese & 8 & 4.80 & \\
\hline Obese ${ }^{* *}$ & 26 & 11.37 & \multirow{2}{*}{$0.001^{*}$} \\
\hline Not obese ${ }^{* * *}$ & 8 & 4.80 & \\
\hline Obese, dyslipidemia & 24 & 10.74 & \multirow{2}{*}{$0.001^{*}$} \\
\hline Not obese** & 8 & 4.80 & \\
\hline Obese, insulin resistance & 9 & 8.85 & \multirow{2}{*}{$0.007^{*}$} \\
\hline Not obese ${ }^{* *}$ & 8 & 4.80 & \\
\hline Obese, dyslipidemia, insulin resistance & 47 & 11.94 & \multirow{2}{*}{$0.003^{*}$} \\
\hline Not obese $\mathrm{e}^{* *}$ & 8 & 4.80 & \\
\hline
\end{tabular}

(*) Mann Whitney Test

$\left({ }^{* *}\right)$ Obese, not dyslipidemia, not insulin resistance

$\left({ }^{* * *}\right)$ Not obese, not dyslipidemia, not insulin resistance

This study revealed a significant difference in the VCAM-1 values of obese adolescents compared to those in non-obese adolescents. The VCAM-1 values in obese adolescents increased 2.4x compared to those in non-obese adolescents.

This study revealed a significant difference in the VCAM-1 values in obese adolescents who had no dyslipidemia and insulin resistance compared to those in non-obese adolescents who had no dyslipidemia and insulin resistance. The VCAM-1 values in obese adolescents who had no dyslipidemia and insulin resistance increased $2.4 \mathrm{x}$ compared to those in non-obese adolescents who had no dyslipidemia and insulin resistance. This study revealed a significant difference in the VCAM-1 values in 
obese adolescents who had dyslipidemia but had no insulin resistance compared to those in non-obese adolescents who had no dyslipidemia and insulin resistance. The VCAM-1 values in obese adolescents who had dyslipidemia but had no insulin resistance increased $2.2 \mathrm{x}$ compared to those in non-obese adolescents who had no dyslipidemia and insulin resistance.

This study revealed a significant difference in the VCAM-1 values in obese adolescents who had insulin resistance but had no dyslipidemia compared to those in non-obese adolescents who had no dyslipidemia and insulin resistance.
The VCAM-1 values in obese adolescents who had insulin resistance but had no dyslipidemia increased $1.8 \mathrm{x}$ compared to those in non-obese adolescents who had no dyslipidemia and insulin resistance.

This study revealed a significant difference in the VCAM1 values in obese adolescents who had dyslipidemia and insulin resistance. The VCAM-1 values in obese adolescents who had dyslipidemia and insulin resistance increased $2.5 \mathrm{x}$ compared to those in non-obese adolescents who had no dyslipidemia and insulin resistance.

TABLE 4: Association Between Dyslipidemia and Insulin Resistance in Obese Adolescents with VCAM-1 Values

\begin{tabular}{lccc}
\hline \multicolumn{1}{c}{ Risk Factors } & n & VCAM-1 (Median) & P $<0.05$ \\
\hline Obese, dyslipidemia & 24 & 10.74 & $0.377^{*}$ \\
Obese** & 26 & 11.37 & $0.174^{*}$ \\
Obese, insulin resistance & 9 & 8.85 & $0.769^{*}$ \\
Obese** & 26 & 11.37 & 11.94 \\
Obese, dyslipidemia, insulin resistance & 47 & 11.37 & \\
Obese** & 26 & & \\
\hline
\end{tabular}

(*) Mann Whitney Test

$(* *)$ Obese, not dyslipidemia, not insulin resistance

This study revealed no significant difference in the VCAM1 values in obese adolescents who had dyslipidemia but had no insulin resistance compared to those in obese adolescents who had no dyslipidemia and insulin resistance.

This study revealed no significant difference in the VCAM-1 values in obese adolescents who had insulin resistance but had no dyslipidemia compared to those in obese adolescents who had no dyslipidemia and insulin resistance.

This study revealed no significant difference in the VCAM1 values in obese adolescents who had dyslipidemia and insulin resistance compared to those in obese adolescents who had no dyslipidemia and insulin resistance.

\section{DISCUSSION}

In this study, 93\% of the subjects were obese adolescents. Most of the subjects in this study were adolescents aged $>15$ - 18 years. In Thailand, the prevalence of obesity in children aged $6-12$ years rose from $12.2 \%$ to $15.6 \%$ over 2 years [13]. The prevalence of obesity in children aged 6-18 years is much higher than in preschool children [14]. Some contributing factors include higher fast-food consumption, lower activity levels, and more frequent TV consumption [15]. Good economic growth, increased economic stability, and lifestyle changes in the Asian region over the last three decades have led to significant improvements in food and nutrition issues in Asian countries [16].

All of the subjects in this study were male. This study analyzed the correlation between obesity, dyslipidemia, and insulin resistance and the VCAM- 1 values. These conditions are the risk factors for endothelial dysfunction [1], [2], [10]. In which the VCAM-1 values will increase [17]. For women, estrogen is among the protective factors against endothelial dysfunction [18], [19].

This study revealed that age, weight, height, and BMI did not correlate with the VCAM-1 values. The sVCAM-1, sICAM-1, and E-Selectin values did not correlate with age in a study conducted in children with the risk of atherosclerosis [20].
There was also no correlation between BMI and VCAM-1 values, in addition to the absence of direct correlation between BMI and ICAM-1 values in a study conducted in children with metabolic syndrome [21]. However, there was an indirect correlation between BMI and ICAM-1 values through HDL (Hye Ah Lee et al., 2020) [21]. This study demonstrated a correlation between increased inflammation and ICAM-1 values [21]. It suggested that endothelial dysfunction may result from chronic inflammation in patients with metabolic syndrome [21]. This study revealed a significant difference in the VCAM$1 \mathrm{~s}$ values of obese adolescents compared to those in nonobese adolescents. The VCAM-1 values in obese adolescents increased $2.4 \mathrm{x}$ compared to those in nonobese adolescents. Obesity is one of the risk factors for endothelial dysfunction. In obese children, there was a decrease in arterial distensibility compared to that in the control group. Arterial endothelial dysfunction occurred in obese children [25], [26], [27], [28].

Obesity may result in low-grade inflammation, increased LDL, increased activity of the renin-angiotensin system, increased concentration of non-esterified fatty acids (NEFA), which can trigger endothelial dysfunction.2 Obesity also may lead to an increase in adipokines which can trigger the formation of new fat cells as well as inflammation that will trigger endothelial dysfunction [29].

Obese children may experience an increased expression of adhesion molecules, including VCAM-1, ICAM-1, and Eselectin, which are among the indicators of endothelial dysfunction [1], [2], [23].

This study revealed a significant difference in the VCAM-1 values in obese adolescents who had no dyslipidemia and insulin resistance compared to those in non-obese adolescents who had no dyslipidemia and insulin resistance. The VCAM-1 values in obese adolescents who had no dyslipidemia and insulin resistance increased $2.4 \mathrm{x}$ compared to those in non-obese adolescents who had no dyslipidemia and insulin resistance. This study observed only the obesity factor, excluding other factors that could cause an increase in VCAM-1, namely dyslipidemia and insulin resistance. It indicates that obesity itself is a factor that causes an increase in VCAM-1 levels. 
Obesity itself is a factor that causes an increase in VCAM1 levels [22], [23]. Obesity may result in low-grade inflammation, increased LDL, increased activity of the renin-angiotensin system, increased concentration of non-esterified fatty acids (NEFA), which can trigger endothelial dysfunction [2], [29].

Obese children accompanied by a family history of cardiovascular disease, smoking, alcohol consumption, sedentary lifestyle, BMI > 85th percentile, experiencing dyslipidemia, insulin resistance, and hypertension have a high risk of atherosclerosis. Therefore, it is necessary to assess and intervene for endothelial dysfunction [30]. Children at high risk of atherosclerosis need several interventions, including not consuming alcohol, not smoking, doing regular exercises, regulating diet, and lowering BMI [30]. Children with BMI > 85th percentile require intervention in the form of a healthy lifestyle. Children with $\mathrm{BMI}>95$ th percentile require obesity therapy [30]. A 5\%-8\% reduction in BMI can improve endothelial dysfunction in obese individuals [29].

This study revealed a significant difference in the VCAM1 values in obese adolescents who had dyslipidemia but had no insulin resistance. The VCAM-1 values in obese adolescents who had dyslipidemia but had no insulin resistance increased $2.2 \mathrm{x}$ compared to those in nonobese adolescents who had no dyslipidemia and insulin resistance. Besides obesity, dyslipidemia is also one of the risk factors for endothelial dysfunction [2], [29], [31]. Dyslipidemia may lead to an increase in total cholesterol, triglycerides (TG), low-density lipoprotein (LDL), and a decrease in high-density lipoprotein (HDL) [32]. An increase in total cholesterol, TG, and LDL will lead to an excess of circulating lipids [31]. Excess lipids in the circulation or tissues is an important independent cause of endothelial dysfunction [31], which can also lead to lipotoxicity. Lipotoxicity is a condition in which excess fat in the circulation and tissues causes endothelial dysfunction [31]. A decrease in HDL will reduce the clearance of LDL from the periphery, which can cause an increase in LDL levels [31]. An increase in LDL will trigger atherosclerotic plaques that lead to cardiovascular diseases [31]. Oxidized LDL is an inhibitor of eNOS activation [31]. Dyslipidemia may also lead to reduced availability of tetrahydrobiopterin (BH4), resulting in endothelial nitric oxide synthetase (eNOS) being "unpaired" and paradoxically leading to an increase in reactive oxygen species (ROS), which also contributes to reduced bioavailability of nitric oxide (NO) and vasoconstriction [31].

This study revealed a significant difference in the VCAM1 values in obese adolescents who had insulin resistance but had no dyslipidemia. The VCAM-1 values in obese adolescents who had insulin resistance but had no dyslipidemia increased $1.8 \mathrm{x}$ compared to those in nonobese adolescents who had no dyslipidemia and insulin resistance. In obesity, apart from chronic inflammation, insulin resistance also plays a role in the development of endothelial dysfunction [2], [33].

Insulin exerts pro and anti-atherogenic effects on blood vessels [33]. In normal endothelial function, in response to stress, stress activates a Phosphatidinositol 3-kinasedependent (PI3K) signal that causes phosphorylation of eNOS [29]. eNOS is required by BH4 to convert L-arginine to L-citrulline and NO [29]. NO will cause smooth muscle relaxation [29]. In conditions of insulin resistance, the endothelium is characterized by decreased signaling in the PI3-K-NO pathway, but the mitogen-activated protein kinase endothelin-1 (MAPK-ET-1) pathway remains the same or the signaling increases.
Therefore, NO stimulation occurs in the endothelium, but endothelin production is not disturbed [29], [33], [34], [35], [36]. The imbalance between NO production and endothelin-1 secretion will cause endothelial dysfunction [29], [33], [34], [35], [36].

Obesity accompanied by insulin resistance also results in increased production of ROS, which causes oxidative stress, which leads to a decrease in NO synthesis [37]. Insulin resistance is also associated with lipotoxicity, glucotoxicity, and inflammation [38]. These conditions will trigger oxidative stress, causing oxidative stress, which leads to a decrease in NO production and results in endothelial dysfunction [34].

This study revealed a significant difference in the VCAM1 values in obese adolescents who had dyslipidemia and insulin resistance. The VCAM-1 values in obese adolescents who had dyslipidemia and insulin resistance increased $2.5 \mathrm{x}$ compared to those in non-obese adolescents who had no dyslipidemia and insulin resistance. Other studies indicate that obesity does not stand alone in causing endothelial dysfunction [2]. Obesity accompanied by other risk factors such as dyslipidemia, insulin resistance poses a higher risk for endothelial dysfunction than that alone in children [2]. In addition to the result of chronic inflammation, endothelial dysfunction in obese children is also caused by dyslipidemia and insulin resistance [23], [29].

Obesity causes fat hypertrophy, which can increase free fatty acids, causing an increase in IL-1, IL-6, IL-18, TNF $\alpha$, and resulting in chronic inflammation [2], [39]. Chronic inflammation will cause an increase in TG, LDL, and a decrease in HDL, leading to dyslipidemia [2] and insulin resistance [39]. These two conditions can trigger oxidative stress [1], [37], leading to endothelial dysfunction [2]. Endothelial dysfunction increases the expression of adhesion molecules, including VCAM-1 and ICAM-1 [1], [2]. However, at the onset of atherosclerosis, VCAM-1 has a more dominant role [11].11

This study revealed no significant difference in the VCAM1 values either in obese adolescents who had dyslipidemia but had no insulin resistance, in obese adolescents who had insulin resistance but had no dyslipidemia, or in obese adolescents who had dyslipidemia and insulin resistance compared to those in obese adolescents who had no dyslipidemia and insulin resistance. The reason for this is that the compared subjects belonged to the obese group. Obesity itself is a factor that causes an increase in VCAM-1 levels [22], [23]. Obesity may result in low-grade inflammation, increased LDL, increased activity of the renin-angiotensin system, increased concentration of nonesterified fatty acids (NEFA), which can trigger endothelial dysfunction [2], [29]. Based on this study, it can be concluded that obese adolescents who had dyslipidemia and insulin resistance significantly associate to an increase in the VCAM-1 values.

\section{REFERENCES}

[1] Halcox JP, Donald AE, Ellins E. Endothelial function predicts progression of carotid intimamedia thickness. Circulation. 2009; 119:1005-12.

[2] Namrata C, Sahiba K, Sahil C, Sarah C, Kavish R. Endothelial Dysfunction in Obese Children. NJLM. 2015; 4:17-24.

[3] Anderson P and Butcher K. Childhood obesity: trends and potential causes. Future Child. 2006; 16:19-45. 
[4] Mahshid D, Noori AD, Anwar TM. Childhood obesity, prevalence and prevention. Nutrition Journal. 2005; 4:1-8.

[5] Kementrian Kesehatan, Republik Indonesia. Riskesdas. 2018:1-200.

[6] Shahara A, Monita H, Suraiya B. Dyslipidemia in childhood obesity: a review. Bangladesh J Child Healt. 2018;42;148-154.

[7] Małgorzata R, Ewelina W, Anna M, Michał, Aneta C, Urszula D, Beata P. Carotid intima-media thickness and metabolic syndrome components in obese children and adolescent. Advs Exp Medicine. 2017:1-10.

[8] António P, Paula M, Ana MP. Insulin Resistance, Dyslipidemia and Cardiovascular Changes in a Group of Obese Children. Arq Bras Cardiol. 2015; 104:266-273.

[9] Ebe D, Ornella G, Francesco C. Atherogenic Dyslipidemia and Cardiovascular Risk Factors in Obese Children. International Journal of Endocrinology. 2015:1-9.

[10] Ranganath $M$ and James RS. Role of Insulin Resistance in Endothelial Dysfunction. Rev Endocr Metab Disord. 2013:14;1-14.

[11] Myron IC, Kaeko I, and Hongmei L. A major role for VCAM-1, but not ICAM-1, in early atherosclerosis. The Journal of Clinical Investigation. 2001; 107:1255-62.

[12] Kyoung-Ha P and Woo JP. Endothelial Dysfunction: Clinical Implications in Cardiovascular Disease and Therapeutic Approaches. Korean Med Sci. 2015; 30:1213-25.

[13] Mo-Suwan L, Junjana C, Peutpaiboon A, Increasing obesity in school children in a transitional society and the effect of the weihght control program. Southeast Asian J Trop Med Public Healt. 1993; 24:590-4.

[14] James PT, Leach R, Kalamara E, Shayeghi M. The Worldwide Obesity Epidemic. Obesity Research. 2001; 9:228-33.

[15] Andy J, Eddy F, Anggraini A. Risk factors for obesity in 6 to 12-year-old children. Paediatr Indones. 2015;55;35-9.

[16] Tee ES. Obesity in Asia: prevalence and issues in assessment methodologies. Asia Pacific J Clin Nutr. 2002; 11:694-701.

[17] Kim F, Pham M, Luttrell I. Toll-like receptor-4 mediates vascular inflammation and insulin resistance in diet-induced obesity. Circ Res. 2007; 100:1589-96.

[18] Bush TL, Barret-Connor E, Cowan LD, Criqui, MH, Wallace RB, Suchindran CM. Cardiovascular mortality and noncontraceptive use of estrogen in women: results from the Lipid Research Clinics Program Follow-Up Study. Circulation. 1987;75: 1102-9.

[19] Mendelsohn ME and Karas RH. Mechanisms of disease: the potential effects of estrogen on the cardiovascular system. N England J Med. 1999; 340:1801-11.
[20] Wojciech W and Jan G. Soluble ICAM-1, VCAM-1 and E-selectin in children from families with high risk of atherosclerosis. International Journal of Molecular Medicine. 2001;7: 181-5.

[21] Hye Ah Lee, Eun JC, Bohyun P, Hwayoung L. The association between metabolic components and markers of inflammatory and endothelial dysfunction in adolescents, based on the Ewha Birth and Growth Cohort Study. Journal Pone. 2020:1-18

[22] Ibrahim KK, Hasnah B, Yoke KY, Sabariah MN. Endothelial Dysfunction in Obesity-Induced Inflammation: Molecular Mechanisms and Clinical Implications. Biomolecules. 2020:1-21.

[23] Skilton and Celermajer. Endothelial dysfunction and arterial abnormalities in childhood obesity. International Journal of Obesity. 2006; 30:1041-9.

[24] Tounian P, Aggoun Y, Dubern B, Varille V, GuyGrand B, Sidi D, et al. Presence of increased stiffness of the common carotid artery and endothelial dysfunction in severely obese children: aprospective study. Lancet. 2001;358: 1400-04.

[25] Aggoun Y, Tounian P, Dabbas-Tyan M, Massih TA, Girardet JP, Ricour C, et al. Arterial rigidity and endothelial dysfunction in obese children. Arch Mal Coeur Vaiss. 2002; 95:631-5.

[26] Watts K, Beye P, Siafarikas A, O'Driscoll G, Jones TW, Davis EA, et al. Effects of exercise training on vascular function in obese children. J Pediatr. 2004;144: 620-5.

[27] Woo KS, Chook P, Yu CW, Sung RYT, Qiao M, Leung SSF, et al. Overweight in children is associated with arterial endothelial dysfunction and intima-media thickening. Int J Obes Relat Metab Disord. 2004;28: 852-7.

[28] Zhu W, Huang X, He J, Mengxia L, Henning N. Arterial intima-media thickening and endothelial dysfunction in obese Chinese children. Eur J Pediatr. 2005; 164:337-44.

[29] Luc B, Vicky Y, Katrien L, José R, Christiaan JV. Childhood obesity related endothelial dysfunction: an update on pathophysiological mechanisms and diagnostic advancements. Pediatric Research. 2016:1-22.

[30] Mirjam M and Nataša MV. Cardiovascular Risk Factors in Children with Obesity, Preventive Diagnostics and Possible Interventions. Metabolites. 2021; 11:1-18.

[31] Jeong-a K, Monica M, Sruti C, and Michael JQ. Role of Lipotoxicity in Endothelial Dysfunction. Heart Fail Clin. 2012;8,589-607.

[32] Shahara A, Monita H, and Suraiya B. Dyslipidemia in childhood obesity: a review. Bangladesh J Child Healt. 2018;42, 148-54.

[33] Ranganath M and James RS. Role of Insulin Resistance in Endothelial Dysfunction. Rev Endocr Metab Disord. 2013;14, 1-14.

[34] Janus A, Szahidewicz-Krupska E, Mazur G, Doroszko A. Insulin Resistance and Endothelial Dysfunction Constitute a Common Therapeutic Target in Cardiometabolic Disorders. Mediators of Inflammation. 2016:1-10. 
[35] Lteif A, Vaishnava P, Baron AD, Mather KJ. Endothelin limits insulin action in obese/insulinresistant humans. Diabetes. 2007:56:728-34.

[36] Kim JA, Montagnani M, Koh KK, Quon MJ. Reciprocal relationships between insulin resistance and endothelial dysfunction: molecular and pathophysiological mechanisms. Circulation. 2006; 113:1888-904.
[37] Francesco P, Sarah C, Francesco C. Role of oxidative stress in endothelial insulin resistance. World J Diabetes. 2015; 6:326-32.

[38] Ouchi N, Parker JL, Lugus JJ, and Walsh K. Adipokines in inflammation and metabolic disease. Nature Reviews Immunology. 2011;11: 85-97.

[39] Rosario $M$ and Isabel A. Chronic inflamation in obesity and the metabolic syndrom. JACC. 2010:1-10. 